\section{Organization of the visual cortex}

SIR - One long-standing question in developmental neuroscience is whether the functional architecture of the visual cortex is epigenetically induced by activitydependent mechanisms or whether it is prespecified by genetic instructions ${ }^{1,2}$. It is widely assumed that dependence on experience and variability of the individual layout of cortical columns are the hallmarks of activity-dependent self-organization. Bonhoeffer and co-workers have challenged the hypothesis that the pattern of cortical orientation columns arises by activity-dependent refinement of geniculocortical connections ${ }^{3,4}$.

Orientation maps in area 18 of cat visual cortex that were forced to form independently for the left and right eye nevertheless exhibit an unexpectedly large degree of similarity. Godecke and Bonhoeffer argued that their results should be viewed as evidence for an innate predetermination of orientation preference, if orientation preference is determined by the pattern of geniculocortical connections at all $^{4}$. Taking an opposing point of view, we point out here that the observed phenomenon can result from activity-dependent mechanisms if not only microscopic mechanisms, but also global constraints imposed by areal borders and retinotopic organization are taken into account.

It has long been suggested that the activity-dependent refinement of geniculocortical connections might follow dynamics analogous to symmetry breaking and pattern formation in physical variation ${ }^{7}$

systems. The qualitative behaviour of pattern-forming systems generally depends on the ratio of system size and characteristic wavelength of the emerging structure ${ }^{5}$. An archetypical example is the Rayleigh-Bénard instability, where convection rolls form in a fluid layer to transport energy from a heated bottom to a cold top. A small container can accommodate only a few rolls (strong confinement), and repetitions of the experiment will lead to identical patterns. In a large container the emerging pattern of rolls will no longer be predictable ${ }^{6}$. The relevant length scale in area 18 is its mediolateral extension (about $3 \mathrm{~mm}$; ref. 7).The characteristic wavelength of orientation columns is approximately 1.3 $\mathrm{mm}$ (ref. 8). Hence the system studied by Bonhoeffer and co-workers represents the strong confinement case. A repetition of the developmental dynamics should then display very similar stationary solutions as long as areal borders are constant. Nevertheless, orientation maps should vary between different animals because the compartmentalization of the cortical sheet in functional areas also exhibits considerable inter-individual

To investigate this suggestion we studied an idealized model for the development of afferent receptive fields under the influence of pattern vision ${ }^{9}$ in cortical areas of different shape. The emergence of orientation maps was simulated using sequences of random stimuli applied to a

Results of simulations of a simple model for the selforganization of orientation maps $^{9}$ in a quadratic $(a, b)$ and a strongly elongated target area $(c, d)$. Pictures show spatial patterns of preferred orientations in a colour-coded representation. Orientation angles $0^{\circ}$, $45^{\circ}, 90^{\circ}, 135^{\circ}, 180^{\circ}$ are mapped on a colour circle: yellow-green-blue-red-yellow. Starting from identical initial conditions and using identical model parameters, the two self-organization processes differed only in the sequence of randomly chosen stimuli. In the first case the maps exhibit differing layouts
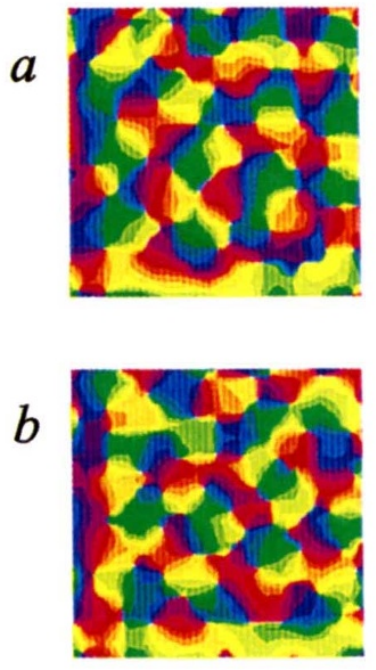
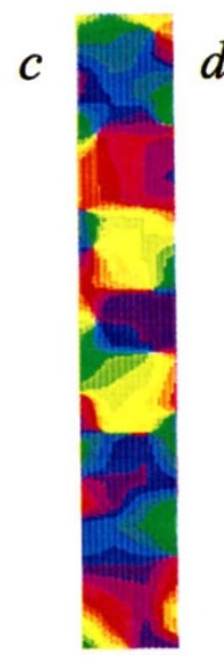

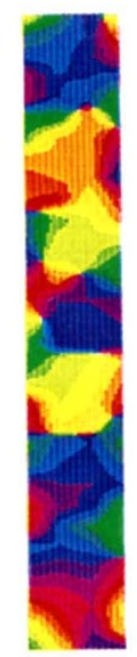

(mean correlation $0.28 \pm 0.05$, evaluated for three pairs of maps); in the second case the maps are virtually identical (mean correlation $0.88 \pm 0.04$, evaluated for three pairs of maps). Maps were generated using Kohonen's self-organizing map algorithm ${ }^{12}$, with $16 \times 16$ (retinal) input channels mapped to $24 \times 24$ map neurons $(a, b)$, and $16 \times 8$ input channels mapped to $36 \times 6$ map neurons $(c, d)$, open boundary conditions. Stimuli were activity distributions of elliptic gaussian shape, length of half-axes $\sigma_{1}=1, \sigma_{2}=5$. Orientation and location of the stimuli were chosen by a numerical pseudo-random procedure, with different random sequences for $a$ and $b$, and $c$ and $d$, respectively. Further parameters of the algorithm were the adaptation step size $\epsilon=5 \times 10^{-5}$, the width of the neighbourhood function $\sigma=1$, and the number of adaptation steps $N=3 \times 10^{6}$. Maps were intialized with pairwise identical, retinotopic connectivity patterns. single model retina. In this system, a reverse-suture experiment should be compared with a pair of simulations starting from identical initial conditions but using different random sequences of stimuli. In areas with large extension in both spatial dimensions, we found maps with only a general structural similarity ( $a, b$ in the figure). In narrow and elongated areas, however, simulations using different sequences of random stimuli led to virtually identical orientation maps ( $c, d$ in the figure). Because variants of this model system have successfully explained various aspects of cortical map formation, and because our simulations clearly show the impact of global geometric constraints on map formation, we conclude that precise restoration of orientation maps in cat area 18 is in fact expected, if orientation maps arise by activity-dependent refinement of geniculocortical connections.

If external boundary conditions actually determine the development of area 18, the results of a reverse-suture experiment might be qualitatively different in a larger area where most columns are distant from areal borders. This is in fact suggested by two reverse-suture studies in area 17 (refs $10,11)$ that are largely inconsistent with the results obtained in area 18. These studies indicate that neurons regaining input from a previously sutured eye exhibit independent orientation preferences in the two eyes.

It might therefore turn out that the phenomenon discovered by Bonhoeffer and co-workers does not represent evidence for an innate predetermination of orientation preference, but demonstrates the influence of global constraints on the formation of neural patterns.

F. Wolf, H.-U. Bauer, K. Pawelzik

\& T. Geisel

Max-Planck-Institut für

Strömungsforschung,

Postfach 2853, D-37018 Göttingen, Germany

and SFB Nichtlineare Dynamik, Universität Frankfurt,

Postfach 111932,

D-60054 Frankfurt, Germany.

e-mail: bauer@chaos.uni-frankfurt.de

BONHOEFFER AND GÖDECKE REPLY Wolf et al. make an interesting suggestion of how to explain our finding ${ }^{4}$ that in cat area 18 similar orientation maps emerge even if the eyes have no simultaneous visual experience.

Rather than contradicting our interpretation, we find that the notion that boundary conditions might determine the structure of orientation maps well in line with the idea that intrinsic or innate factors, rather than visual experience, control the exact layout of cortical orientation maps. In our paper we did not propose that activity-dependent mechanisms are 